\title{
Proteomic profile of acute myeloid leukaemia: A review update
}

\author{
Amer Abdulrahman Almaiman ${ }^{1,2 *}$, Rasedee Abdullah ${ }^{1}$, Ahmad bin Abdul ${ }^{1}$, \\ Zeenathul Allauddin ${ }^{1}$, Eltayeb EM Eid ${ }^{3}$ and Sultan Ayesh Mohammed Saghir ${ }^{4}$ \\ ${ }^{1}$ Institute of Bioscience, Universiti Putra Malaysia, Selangor, ${ }^{2}$ Community College, Applied Medical Sciences, ${ }^{3}$ Uniazah College \\ of Pharmacy, Qassim University, Qassim, Saudi Arabia, ${ }^{4}$ School of Pharmaceutical Sciences, Pharmacology Department, \\ University Sains Malaysia, Penang, Malaysia
}

${ }^{\star}$ For correspondence: Email: ameralmeman@hotmail.com; Tel: +966533544424

\begin{abstract}
Proteome analysis is a complex and dynamic process that encompasses several analytical platforms that include protein sequencing, structural or expression proteomics, protein modification, sub-cellular protein localization, protein-protein interaction and biological functional proteomics. In fact, expression proteomics is extensively applied in a majority of biomarker detection studies because it provides a detailed overview of differentially expressed proteins in cellular pathways and disease processes. Proteomics are also effective and dynamic in protein-protein interactions and cross-talks between interacting molecules of the cell. Proteomics has evolved into a crucial tool used to investigate the biochemical changes that possibly lead to development of cancer biomarkers. This review draws attention to the progress and advancements in cancer proteomics technology with the aim of simplifying the understanding of the mechanisms underlying the disease and to contribute to detection of biomarkers in addition to the development of novel treatments. Given that proteome is a dynamic entity of cellular functions in health and disease, it is capable of reflecting the immediate environmental state of cells and tissues as shown in this review. The review shows the possibility of elucidating the pathophysiology of acute myeloid leukaemia (AML) through proteome expressions, thus confirming the viability of proteome analysis in profiling AML.
\end{abstract}

Keywords: Acute myeloid leukaemia, Proteomics, Expression profile, Protein sequencing, Proteinprotein interaction, Biomarkers

Tropical Journal of Pharmaceutical Research is indexed by Science Citation Index (SciSearch), Scopus, International Pharmaceutical Abstract, Chemical Abstracts, Embase, Index Copernicus, EBSCO, African Index Medicus, JournalSeek, Journal Citation Reports/Science Edition, Directory of Open Access Journals (DOAJ), African Journal Online, Bioline International, Open-J-Gate and Pharmacy Abstracts

\section{INTRODUCTION}

Acute myeloid leukemia (AML) is an invasive hematological cancer that affects different clonal hematopoietic cells leading to distinct differences in their response to chemotherapy treatment [1]. Despite advancement in leukemia therapy, the mortality rate remains high as a result of disease progression. In 2012, United States recorded deaths of 10,200 out of 13,780 AML cases (mortality rate of $74 \%$ ) with majority of the patients unable to withstand chemotherapy [2]. This is due to the fact that hematopoietic stem cells (HSCs) in AML persist in an immature state because their development or differentiation is impeded at the level of progenitor cells resulting in malignant production and accumulation of leukemic cells [3].

Malignant cancer cells frequently exhibit abnormal expression of metabolic and signaling pathways of proteins [4]. Therefore, the classification of AML depends on morphological, 
cytochemical and immunophenotypic cellular characteristics. These cellular characteristics are defined by the molecular entities, which could be targeted for the development of diagnostic and prognostic profiles, and for the identification of disease subtypes [5]. The genome and their respective proteins have recently become valuable tools in hematology research. It is established that genetic changes result in altered cellular processes, which in turn affect differentiation, growth control and induce apoptosis. Proteins are workforce and thrust for nearly every cellular metabolic processes and serve in the functional mechanism for cells, tissues or organs [6].

Recent advancements in proteomic technologies have garnered immense interest in the search for biomarkers for different hematological malignancies. Comprehensive analysis of proteins from cells, tissues, fluids, or organs, using the classical proteomic platforms has provided data on the disease heterogeneity at different phases of growth and progression. Therefore, proteomics are currently increasingly being sought in cancer research to characterize abnormally expressed proteins that could potentially be used as disease biomarkers [6] These biomarkers would complement the more conventional diagnostic techniques such as cytogenetics, hematology, and other clinical tests to effectively diagnose and offer prognosis of $A M L$, in addition to minimizing the use of invasive procedures like bone marrow and tissue biopsies $[7,8]$.

\section{Synopsis of proteomics techniques}

Proteomes are the outcomes of the expression of thousands of genes. The complexities associated with proteome identification can be attributed to the presence of posttranslational modifications (PTMs) and large variation in dynamic ranges. Therefore, simplifying the mechanism of proteomes would increase the efficiency of the analysis. Accordingly, a considerable part of proteomic research is devoted to the improvement of separation techniques in order to better resolve complex proteomes [9, 10]. Regardless of the advancement in liquid chromatography, there remains the need to study proteomics comprehensively (top-down and bottom-up proteomics) to improve the understanding of cell biology [11]. This is inevitable because it is very difficult to depend on one method or equipment to identify and quantify components of a complex protein sample in a simple, single-step operation. Several tools and procedures are required in separation and identification of polypeptides in addition to integrating and analysis of data.

The major separation tools used in proteomic identification comprise gel-based proteomics, ionization methods, mass spectrometry tools, and quantification approaches [12]. The twodimension electrophoresis (2-DE) is based on separation of proteins on two independent parameters of charge and molecular mass in an electric field and it is the most efficient and highresolution protein separation method for complex mixture of samples. These methods are presently well-optimized and are regularly applied in proteomics analysis $[13,5]$. Proteomic mass spectroscopy-based platforms are used to identify large numbers of novel proteins with the potential to be biomarkers. The strategies of proteomic biomarkers involve the identification of markers using multidimensional protein identification technology coupled with mass spectroscopic identification [14]. Fluorescence in situ hybridization (FISH) is used to monitor diseases and identify specific abnormalities. The FISH probes the following abnormalities: $t$ $(15 ; 17)$ translocation, $t(8 ; 21)$ translocation, inversion (16), 11q23,+8, +21, +22 del(7q), which are used to identify prognostically important translocations in newly diagnosed AML [15].The current usage of computational systems to analyze data related to biomolecules on a largescale platform has become a crucial and firmly recognized discipline in molecular biology. Bioinformatics science has become vital to biological research because of its use in managing large volume of records and deciphering the complex dynamic process [16].

\section{Methods}

The strategy adopted in this study is to search for the related articles archived in the Science Direct database using selected key words, specifically, 'acute myeloid leukaemia' and 'proteomics'. The database was searched for papers published between February, 2012 and February, 2016. This review only cited the available research articles related to AML and it does not include books, conference proceedings and reviews. The studies were represented in a descending order from 2016 to 2012.

Generally, most of the studies exploring AML were done using AML patient samples or cell lines and few studies used aptamer and retrospective analysis. As depicted in Figure 1, 8 articles comprising around $44.4 \%$ of total publications investigated AML using human samples, while 7 articles (38.9\%) used cell lines. 


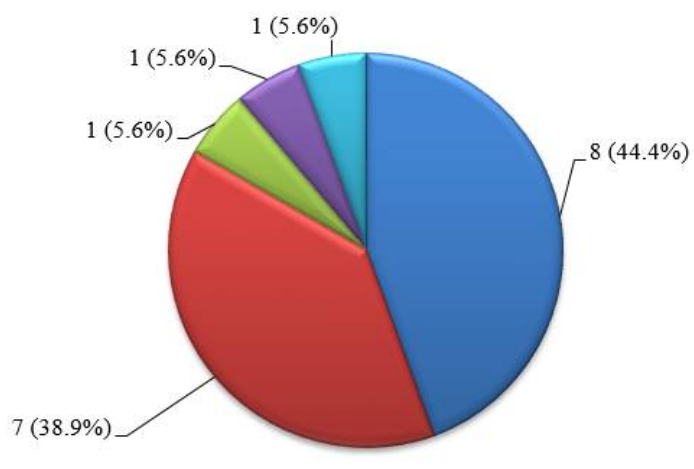

$\square \mathrm{AML}$ pateints $\square$ cell lines $\square$ Aptamer $\square \mathrm{HAPD} \square \mathrm{RA}$

Figure 1: Numbers and percentages of publications related to acute myeloid leukaemia from February 2012 till February 2016 classified according to type of sample used. HAPD; high abundance protein depletion, RA; retrospective analysis

\section{Review of studies highlighting proteomic profiles of acute myeloid leukaemia}

Studies exploring the proteomic profiles of AML from 2016 to 2012 are summarized in Table 1a and $1 \mathrm{~b}$ according to the Science Direct website. It summarizes the aims of previous studies, methods used in investigation, sample number, and sample type.

In a recent study, the association between BCL11A expression at diagnosis and clinical outcomes in newly-diagnosed persons suffering from AML was assessed. They explicitly used reverse transcriptase and real-time polymerase chain reaction (RT-PCR) to determine levels of BCL11A mRNA in bone marrow and blood mononuclear cells in 292 consecutive newlydiagnosed subjects affected with AML. The data was afterward comparatively analyzed against mRNA levels in bone marrow cells of normal individuals. TheBCL11A transcript levels of subjects at the time of diagnosis exceeded the median value of $2.434( \pm 3.423 \mathrm{SD}$; 25th -75 th inter-quartile range, $1.33-4.29$ ). White blood cell (WBC) levels was found to be high and the main fraction of bone marrow myoblasts were classified as FAB MO subtype and less possibility of being $F A B M 3$ subtype, and is thus more prone to be in the intermediate cytogenetic risk cohort and less likely to have a complex karyotype and more likely to have DNMT3AR882 and FLT3-ITD mutations than subjects with transcript levels below the median value. 89 persons were subjected to conventional induction chemotherapy. The complete remission rate in the 89 subjects was reported to be $54 \%$ with 95 $\%$ confidence interval (Cl); 33, $75 \%$ ) in the lower BCL11A cohort and $65 \%(45,85 \%,(p=0.26)$ in the higher BCL11A group. 3 year survival was 33 $\%(2,65 \%)$ in the lower BCL11A cohort and 15 $\%(0,39 \%,(p=0.35)$ in the high BCL11A cohort. There is a considerable relationship between the transcript levels of BCL11A during diagnosis with several clinical and laboratory variables. While, no significant correlation was detected between complete remission rate and survival. These data indicate that BCL11A expression possibly plays role in AML [1].

Currently, the clinical outcomes of 51 AML patients who were subjected to transplantation were evaluated using a standardized reducedintensity conditioning treatment that incorporates post-transplantation azacitidine (AZA). They discovered that 14 patients did not initiate AZA either because of extraction of permission or transplantation impediments. It was noted that 37 patients initiated AZA for durations ranging from 40-194 days (median of 54 days) after transplantation. 37 patients completed 3 or more cycles of AZA, while 16 patients relapsed 8 months (median time) after transplantation. No patient was observed to develop widespread chronic graft-versus-host disease. It was thus inferred that the AZA is well accepted after transplantation and appears to have the potential to lower the relapse risk in patients who display a CD8+ $T$ cell response to tumor antigens [17].

A study was carried out to assess the epidemiological profile and the chances of survival of AML patients in a Hospital Infantil Joana de Gusmão. 51 patients diagnosed with AML between 2004 and 2012 were included in this study. Of the 51 patients studied, $84 \%$ were white; $45 \%$ were females and $55 \%$ were males. In addition, $8 \%$ of the patients were below 1 year, $47 \%$ were aged between 1 and 10 years, and $45 \%$ were older than 10 years. The major symptoms reported for the patients were fever $(41.1 \%)$, asthenia $(35.2 \%)$, and hemorrhagic manifestations $(27.4 \%)$. The central nervous system was the most affected extra-medullary site $(14 \%)$. Diagnosis showed WBC count of below $10,000 / \mathrm{mm}^{3}$ in $47 \%$ of patients. The minimal residual disease (MRD) was determined to be $<0.1 \%$ on the 15 th day of treatment in 16 $\%$ of the sample. Medullary relapse arose in 14 $\%$ of cases. Comparative analysis between the bone marrow MRD and the vital status showed that $71.42 \%$ and $54.05 \%$ of the patients with type M3 AML and non-M3 AML, respectively, were alive. Mortality rate of $43 \%$ was then reported, attributable to septic shock (63.6\%) [18]. 
In a different study, 2-DE-based proteomics was used to investigate cisplatin-induced effects in vitro of human liver cells (L02). The major objective of the study was to identify proteomic responses, which were subsequently used as endpoints to determine the dose responsive effects of cisplatin in human liver L02 cells in three different concentrations $(1 \mathrm{nM}, 1 \mathrm{M}$ and 1 $\mathrm{mM})$. The results of this study suggest that instabilities in protein synthesis and oxidative stress by differential mechanisms were induced due to the different cisplatin treatments. Given that the experimental concentrations of cisplatin exhibit a hormesis effect in cell proliferation of L02 cells, proteomic markers are expected to denote this hormesis effects. A hormesis effects with a U-shape regulation were discernible from up-regulation of confilin-1 among the three concentrations used in cisplatin treatments. The results are consistent with toxico-proteomic studies, which pointed out that the toxicoproteomic responses based on dose-dependent protein responses are incongruent with the theoretically linear or hormetic concentrationeffect relationship. These findings suggest that the proteomic responses induced by cisplatin treatments do not have the ability to reflect a macroscopic hormesis phenomenon on cell proliferation [19].

Last study was carried out using different elution conditions to improve the recovery of all classes of proteins and maintain their compatibility with immunoblotting techniques. The use of double elution and formic acid combined with urea was found to be the most efficient and commonly applicable elution method for chemical proteomics [20]. Also, the prognostic role of AKT mediated GSK3 phosphorylation in AML was examined to explore the GSK3 $\alpha / \beta$ phosphorylation using reverse phase protein analysis (RPPA) in a group of 511 AML patients [21]. They found a positive correlation between levels of phosphorylated GSK3 versus patient survival and expression of other proteins in AML cell survival. The study also showed that high levels of $p-G S K 3 \alpha / \beta$ are correlatable with adverse general survival and a lower occurrence of complete relapse duration in patients with intermediate cytogenetics. In contrast, no correlation was observed in those with unfavorable cytogenetics. Intermediate cytogenetic patients with FLT3 mutation showed a correlation at low rates of $p-G S K 3 \alpha / \beta$. Phosphorylated GSK3 $\alpha / \beta$ expression was evaluated against that of 229 related cell cycle arrest and/or apoptosis proteins [21].
RPPA showed that $p-G S K 3 \alpha / \beta$ is positively correlatable with phosphorylation of AKT, BAD, and P70S6K, but negatively correlated with $\beta$ catenin and FOXO3A. In addition, the positive correlation between PKC $\delta$ and $p-G S K 3 \alpha / \beta$ expression suggest a crosstalk between the AKT and PKC signaling pathways in AML cells. It was inferred from their findings that AKT-mediated phosphorylation of $G S K 3 \alpha / \beta$ may promote the survival of AML cells, and thus damaging to the overall survival of AML patients. Therefore, $p$ GSK3 $\alpha / \beta$ basically serves as a significant adverse prognostic factor for a subset of AML patients [21].

In another study, the safety, maximum tolerated dose (MTD), and dose-limiting toxicity (DLT) of ruxolitinib at doses of 50,100 , and $200 \mathrm{mg}$ b.i.d. in patients with relapsed/refractory AML were evaluated. Twenty seven patients older than $14 \mathrm{y}$ (26 relapsed or refractory AML and 1 patient was affected by acute lymphoid leukaemia) were signed up in this study. It was deduced that the patient with multiple relapses and incomplete recovery of peripheral blood counts was subjected to 7 lines therapy [6].

Furthermore, the levels of expression of TRIM62 protein in $511 \mathrm{AML}$ patients were explored using reverse-phase protein array technology. It was observed that low levels of TRIM62 are related to markedly poorer outcomes but enhances the prognostic impact of Nucleophosphamin1(NPM1) and FLT3 mutations. Therefore, low level of TRIM62 is an independent adverse prognostic factor in AML consistent with a tumor inhibiting role [22]. Similarly, kinase activity profiling was used to recognize collaborators of Spleen Tyrosine Kinase (SYK) in AML. It was observed that FMS-like tyrosine kinase 3 (FLT3) is trans-activated by SYK via direct binding. FLT3-ITD positive AML presented highly activated SYK which binds to FLT3-ITD to activate MYC transcriptional programs. SYK was highly suppressed with FLT3-ITD AML cells rather than FLT3 wild-type complements. It was thus inferred that in vivo model FLT3-ITD, SYK is critical for myeloproliferative disease (MPD) development. Over expression of SYK facilitates transformation to AML and resistance to FLT3ITD-targeted therapy [23].

Studies were also performed to chemically adapt oligonucleotide aptamers with different functional molecules and it can bind specifically with biomarkers on cancer cells to be used in treating AML $[24,25]$. To target AML cells, a single-strand DNA aptamer specific for CD117 biomarker was 
developed. Sequence alignment exhibited that the aptamer contained a G-rich core region with a well-conserved functional G-quadruplex structure [26]. Functional assays demonstrated that this synthetic aptamer was able to specifically precipitate CD117 proteins from cell lysates, selectively bound cultured and patient primary AML cells with high affinity $(K d<5 \mathrm{nM})$, and was specifically internalized into CD117expressing cells [26]. Exposure of patient marrow specimens to aptamer-methotrexate (Apt-MTX) resulted in selective growth inhibition of primary AML cells and had no toxicity to off-target background normal marrow cells within the same specimens. The result of this study showed that the Apt-MTX presented a potential clinical value for AML therapy with minimal to no side effects in patients. The Apt-MTX opens an avenue to chemical synthesis of new targeted biotherapeutics [26].

An earlier study investigated the proteome analysis of four AML cell lines (NB4, THP1, K562 and HL60), one chronic myeloid leukaemia $(\mathrm{CML})$ cell line and of granulocytes isolated from normal human peripheral blood [27]. 823 proteins were identified and relatively quantified using cell surface biotinylation, followed by capture on streptavidin resin, tryptic digestion and Deep QuanTR-assisted mass spectrometric analysis. CD166 or Activated Leukocyte Cell Adhesion Molecule (CD166/ ALCAM) protein was studied at length, because it has been proven to be strongly up-regulated in all AML cell lines, compared to CML and granulocyte controls [28]. More than 100 identified proteins (such as CD33, CD166, integrin alpha-4 and embigin) were found to be relatively more up-regulated in the AML cell lines compared to granulocytes. In addition, the duocarmycin derivatives were found to be more effective compared to the microtubule-targeting cemadotins and maytansinoids. The outcomes of the study were consistent with fluorescenceactivated cell sorting (FACS) validation experiments which could be developed into the basis for antibody-based drug development programs which target human leukaemia [27].

In addition, the expressions of miRNA profiles of cell lines were studied along with differential expression analysis and target prediction of numerous miRNA signatures in both $A M L$ and CML cell lines [29]. The miRNome of two human AML cell lines (HL-60 and THP-1) and one human CML cell line (K-562) were analyzed using vastly parallel signature sequencing. Some miRNAs may possibly act through tumor suppressors or oncomiRs contained in AML and
CML cell lines by targeting key genes in AML and CML pathways. Thus, characteristics of K562, HL-60 and THP-1 cell lines such as actin filament-based processes, reaction to stimulus and phagocytic activity can be evaluated via expression patterns of cell type-specific miRNAs. It was also recommended that miRNA scan regulate myeloid differentiation, since they generally inhibit differentiation regulators. The study provided a basis to further study the use of miRNAs in human leukaemia subtyping, leukemogenesis and myeloid development. In addition, the unique miRNA signatures were suggested as potential candidates for the clinical diagnosis, prognosis and treatment of myeloid leukaemia [29].

Likewise, the potential mechanism essential to the regulation and expression of B55a, in addition to the likely role of the $B$ subunit as a tumor suppressor in AML were comprehensively studied [30]. A strong correlation was observed between B55 $\alpha$ and several proteins (including MYC, PKCa, and SRC) after reverse phase protein analysis (RPPA) of 230 proteins in 511 AML patients. B55a suppression in OCl-AML3 cells by shRNA indicated that the $B$ subunit is a PKCa phosphatase. Rather than target SRC, the kinase (B55a) suppresses protein expression of the $B$ subunit. Lastly, the correlation between $B 55 \alpha$ and MYC levels reflected a complex stoichiometric competition between $B$ subunits. Loss of B55a in OCl-AML3 cells did not change global PP2A activity and only the isoform containing B56a is induced. In cells containing B55a shRNA, MYC was suppressed with simultaneous induction of the competing $B$ subunit B56a (PPP2R5A). A current study also determined that FTY-720, a drug whose mechanism entails the activation of PP2A, resulting in the induction of B55a in AML cells, and $a$ reduction of the $B$ subunit that renders these cells resistant to FTY-720 increase the expression of miR-191-5p and a suppression of miR-142-3pcan be attributable to reduction of the $B$ subunit. Interestingly, miRs were greatly regulated by $B 55 \alpha$ and a poor survival in AML was indicated by high levels of miR-191. The miR-142-3p was mutated in approximately $2 \%$ of AML patients. Conclusively, the suppression of B55a activates signaling pathways that could guarantee the survival of leukaemia cells [30]. The validity of applying the proteomic profiling would require comprehensive verification via clinical trials on mice or by using advanced and sensitive molecular assays such as western plot (WP) or RT-PCR. To overcome some of the aforementioned problems, particularly in the 
biomarker discovery phase, genetically stable models of mice with the disease would simplify the initial use of protein biomarker for candidate selection [31].

A comparative study was done to analyze the efficacies of three high-abundance protein removal methods using 2-DG. The studied methods include the ProteoPrep $®$ Blue Albumin and IgG Depletion Kit (Sigma-Aldrich, USA), the ProteoPrep® Immunoaffinity Albumin and IgG Depletion Kit (Sigma-Aldrich, USA), and the Albumin and IgG Depletion SpinTrap Kit (Amersham Biosciences, Sweden). Their study showed that high-abundance proteins were removed with greatest efficiency using the ProteoPrep $®$ Immunoaffinity Albumin and IgG Depletion Kit and the Albumin and IgG Depletion SpinTrap Kit[32]. An earlier study highlighted that majority of AML patients who tolerate the treatment were able to attain complete remission after chemotherapy, although these patients relapse a few years after remission [33].

The majority of proteomic studies on bone marrow and peripheral blood cells in AML were only performed at time of diagnosis. Hence, not much is known about the proteomic profile of the bone marrow and peripheral blood cells at remission or relapse [2], although, one study attempted to comparatively analyze AML at diagnosis, following remission, and at recurrence. No significant difference was observed in the mononuclear cell proteomic profiles between AML patients at diagnosis and recurrence. Therefore, it was suggested that the proteome expressions of cells before treatment and at relapse are similar, in any case at the level of high concentration of proteins [2]. In 2012, one study also carried out by Luczak et al to identify proteins in the bone marrow and peripheral blood samples collected from healthy subjects and patients with AML-M1/M2. 25 proteins demonstrated differential expression. Based on the findings, the AML samples were readily grouped into M1 and M2 AML. However, only the M2 AML group showed expression of 6phosphogluconate dehydrogenase, L-plastin, and Annexin III [3].

Quantitative phosphoproteomics was investigated in order to recognize gefitinib- and erlotinib stimulated transformations on the level of site-specific serine, threonine and tyrosine phosphorylation in AML cells [34]. The affinities of direct AML cell targets for both kinase inhibitors were evaluated using quantitative chemical proteomics. SILAC-based quantitative mass spectrometry was applied to examine 10,975 distinct phosphorylation sites to characterize the phosphoproteome of KG1 AML cells and its regulation upon erlotinib and gefitinib treatment. It was reported that less than 50 sitespecific phosphorylations were considerably altered, exhibiting specific interference with AML cell signaling. The data proposed that cellular distortion of SFKs and/ or Btk transform into rather specific signal transduction inhibition, which induces the antileukaemic activity of erlotinib and gefitinib in AML, respectively [34].

2-DGsystems and MALDI-TOF/MS were used to study the protein expression in AML1/ETO positive cells in order to illustrate the mechanism of azacitidine (AZA) and decitabine (DAC), in addition to determining their biological activity in Kasumi-1 cells [35]. Cyclophilin A, catalase, nucleophosmin and PCNA were decreased exclusively by azacitidine only, while TCP 1 and hnRNP A2/B1 were decreased by both drugs (AZA and DAC). Alpha-Enolase and Peroxiredoxin-1 showed decreased after treatment with decitabine. Of interest, is the fact that Cyclophilin A was also proposed as a marker of cell necrosis, which was stimulated by decitabine [35]. AZA and DAC displayed diverse mechanisms consistent with their activities on cell viability, protein synthesis, cell cycle, miRNA and gene expression [36-38]. AZA and DAC exhibit specific and discriminatory modulations of the target proteins which are in agreement with the proposed theory which hypothesizes that these two agents exhibit different mechanisms. Despite the dissimilarity between AZA and DAC, they can be used in clinics in alternating or combined ways [35].

\section{CONCLUSION}

Proteomics is an obvious approach for profiling acute myeloid leukaemia because proteins change with cellular responses to internal environment, external stimuli, and disease development. The quest to transform basic discoveries into patient care regimes has resulted in several proteomic studies being carried out to profile potential biomarkers. However, it is disappointing that despite the technological advancement in the field, very few studies have manifested into clinical trials. It is thus vital that polypeptides identified as potential biomarkers are validated in suitably processed and well-characterized clinical samples using recognized techniques such as immunochemistry or PCR-based methods as pointed out in the review. 
Table 1: Summary of studies investigating proteomic profiles of acute myeloid leukaemia

\begin{tabular}{llll}
\hline Target & Used method & Sample & Reference
\end{tabular}

Study the associations between BCL11A expression at diagnosis and clinical Assessment of AML patients subjected to transplantation

Investigating the epidemiological profile and the survival rate of AML patients

Investigate cisplatin-induced effects in vitro of human liver cells (L02).

Improve the recovery of all classes of proteins and maintain their compatibility with immunoblotting techniques

Study the prognostic role of AKT mediated GSK3 phosphorylation in AML

Evaluation of the safety, MTD and DLT of ruxolitinib patients with relapsed/refractory AML

Exploring the levels of expression of TRIM62 protein

Identification of collaborators of Spleen

Tyrosine Kinase (SYK) in AML

Develop new therapeutics targeting AML

Investigate the proteome analysis of four AML cell lines (NB4, THP1, PLB985, and HL$60)$ and one CML (K-562)

Study the expression of miRNA profiles of two human AML cell lines (HL-60 and THP1 ), and one human CML cell line (K-562) followed by differential expression analysis and target prediction

Investigation of the potential mechanism underlying the regulation and expression of $\mathrm{B} 55 \mathrm{\alpha}$, in addition to the possible role for the B subunit as a tumor suppressor in AML

Comparison between the efficiency of three high-abundance protein depletion methods

\section{Rt\& RT-PCR}

SRIC incorporating post-transplantation $A Z A$

clinical-epidemiological, $51 \mathrm{AML}$ patients observational,

retrospective, descriptive study

2-DE

Gel-free liquid chromatography coupled with mass spectrometry (LCMS) GSK3 $\alpha / \beta$ phosphorylation by RPPA

A single-center phase I/II clinical study testing 3 dose levels $(50 \mathrm{mg}$ $(\mathrm{n}=4), 100 \mathrm{mg} \quad(\mathrm{n}=5)$ and $200 \mathrm{mg}(\mathrm{n}=18)$

Reverse-phase protein array technology kinase activity profiling

A single-strand DNA aptamer specific for CD117 biomarker

Cell surface

biotinylation and mass spectrometric analysis

Massively parallel sequencing for an indepth analysis of the miRNomes

RPPA of 230 proteins

511 AML patients

SDS-PAGE and 2-DG

ProteoPrep Blue Albumin and IgG Depletion Kit, ProteoPrepImmunoaffinity Albumin and IgG Depletion Kit and Albumin and IgG Depletion SpinTrap Kit
[1] 
Table 1: Summary of studies investigating proteomic profiles of acute myeloid leukaemia (Continued)

\begin{tabular}{|c|c|c|c|}
\hline Target & Used method & Sample & Reference \\
\hline $\begin{array}{l}\text { Compare the modalities of MRD detection in } \\
\text { AML }\end{array}$ & $\begin{array}{l}\text { Discussing recently } \\
\text { reported clinical trials }\end{array}$ & & [33] \\
\hline $\begin{array}{l}\text { Compare between proteome expression of } \\
\text { cells prior to treatment and at relapse }\end{array}$ & $\begin{array}{l}\text { 2-DG, Gel image } \\
\text { analysis and mass } \\
\text { spectrometry }\end{array}$ & 38 AML (M1 or M2) patients & [2] \\
\hline $\begin{array}{l}\text { Investigation of the quantitative } \\
\text { phosphoproteomics to identify gefitinib- and } \\
\text { erlotinib induced changes on the level of site- } \\
\text { specific serine, threonine and tyrosine } \\
\text { phosphorylation in AML cells }\end{array}$ & $\begin{array}{l}\text { SILAC-based } \\
\text { quantitative mass } \\
\text { spectrometry }\end{array}$ & $\begin{array}{l}\text { 10,975 distinct } \\
\text { phosphorylation sites for } \\
\text { characterization of the KG1 } \\
\text { AML cells phosphoproteome }\end{array}$ & [34] \\
\hline $\begin{array}{l}\text { Examine the protein expression in } \\
\text { AML1/ETO positive cells to illustrate the } \\
\text { mechanism of action of azacitidine and } \\
\text { decitabine, as well as to determine their } \\
\text { biological activity }\end{array}$ & $\begin{array}{l}\text { 2-DG system, followed } \\
\text { by MALDI-TOF/MS }\end{array}$ & Kasumi- 1 cells & [35] \\
\hline
\end{tabular}

SRIC; Standardized Reduced-Intensity Conditioning, AZA; Azacitidine , Rt; Reverse Transcriptase, RT-PCR;Real Time-Polymerase Chain Reaction, 2-De; Two-Dimensional Electrophoresis, RPPA; Reverse Phase Protein Analysis, MTD; Maximum Tolerated Dose, AML; Acute Myeloid Leukaemia, Apt; Aptamer, MTX; Methotrexate, CML; Chronic Myeloid Leukaemia, HL-60; acute promyelocytic leukemia, THP-1; Acute Monocytic Leukemia, PLB985; acute myeloid leukemia (derivative of HL-60) K-562; Chronic Myleoid Leukaemia, Kasumi-1; Human Acute Myeloid Leukemia, NPM; Nucleophosphamin, MDS; Myelodysplastic Syndrome, KG1; acute myelogenous leukemia, LCMS; Liquid Chromatography Mass Spectrometry

This is important given that understanding the molecular mechanisms in treatment responses and disease recurrence is limited in AML. The majority of obtainable biomarkers are single biomolecules with low sensitivity and specificity. Therefore, the use of a combination of measurable protein panels for a more accurate diagnosis and prediction of disease prognosis in AML is highly recommended based on the review. Proteome analysis was also shown to form a link between gene expressions and cellular function, which can be used afterwards to evaluate disease growth, prognosis and reaction to treatment. Moreover, by mapping the location of proteins in cells (proteome analysis); it is possible to examine molecular and biological mechanisms. Based on the review and the inherent benefits of proteome analysis, this paper suggests its use in viably profiling acute myeloid leukaemia.

\section{DECLARATIONS}

\section{Acknowledgement}

The authors highly appreciate Universiti Putra Malaysia, Institute of Bioscience, Malaysia and Al-Qassim University, Unaizah College of Pharmacy, Qassim, Saudi Arabia for their support.

\section{Conflict of Interest}

No conflict of interest associated with this work.

\section{Contribution of Authors}

The authors declare that this work was done by the authors named in this article and all liabilities pertaining to claims relating to the content of this article will be borne by them.

\section{REFERENCES}

1. Tao H, Ma X, Su G, Yin J, Xie X, Hu C, Liu H. BCL11A expression in acute myeloid leukaemia. Leuk Res 2016; 41: 71-75.

2. Pemmaraju N, Kantarjian H., Kadia T., Cortes J, Borthakur G, Newberry K, Pierce S. A phase I/II study of the janus kinase (JAK) 1 and 2 inhibitor ruxolitinib in patients with relapsed or refractory acute myeloid leukemia. Clin Lymphoma Myeloma Leuk 2015; 15(3): 171-176.

3. Luczak M, Kazmierczak M, Handschuh L, Lewandowski K, Komarnicki M, Figlerowicz M. Comparative proteome analysis of acute myeloid leukaemia with and without maturation. Proteomics 2012; 75: 5734-5748.

4. Boyd RS, Dyer MJ, Cain K. Proteomic analysis of b-cell malignancies. Proteomics 2010; 73: 1804-1822.

5. Bacher U, Kohlmann A, Haferlach C, Haferlach T. Gene expression profiling in acute myeloid leukaemia. Best Pract Res Clin Hematol 2009; 22:169-180. 
6. Alaiya AA, Al-Mohanna $M$, Aslam M, Shinwari Z, AlMansouri L, Al-Rodayan M, Al-Eid M, Ahmad I, Hanash K, Tulbah A, Bin Mahfooz A, Adra C. Proteomics-based signature for human benign prostate hyperplasia and prostate adenocarcinoma. Int J Oncol 2011; 38: 10471057.

7. Cui JW, Wang J, He K, Jin BF, Wang HX, Li W, Kang LH, Hu MR, Li HY, Yu M. Proteomic analysis of human acute leukaemia cells: insight into their classification. Clin Cancer Res 2004; 10: 6887-6896.

8. Monteoliva L, Albar JP. Differential proteomics: an overview of gel and non-gel based approaches. Brief Funct Genomic Proteomic 2004; 3: 220-239.

9. Issaq HJ, Conrads TP, Janini GM, Veenstra TD. Methods for fractionation, separation and profiling of proteins and peptides. Electrophoresis 2002; 23: 3048-3061.

10. Issaq HJ. The role of separation science in proteomics research. Electrophoresis 2001; 22: 3629-3638.

11. Speicher D. Overview of proteome analysis. Proteome Analysis. Interpreting the Genome. Elsevier, BV, 2004.

12. Ravandi F, Jilani I, Estey E, Kantarjian H, Dey A, Aguilar C, Albitar M. Soluble phosphorylated fms-like tyrosine kinase: III. FLT3 protein in patients with acute myeloid leukemia. Leuk Res 2007; 31: 791-797.

13. Magdeldin S, Enany S, Yoshida Y, Xu B, Zhang $Y$, Zureena Z, Lokamani I, Yaoita E, Yamamoto T. Basics and recent advances of two dimensional-polyacrylamide gel electrophoresis. Clin Proteomics 2014; 11: 16-18.

14. Zolg JW, Langen $H$. How industry is approaching the search for new diagnostic markers and biomarkers. Mol Cell Proteomics 2004; 3: 345-354.

15. Mehta P. ARUP Consult: The physician's guide to laboratory test selection and interpretation. JAMA 2012; 307: 2436-2437.

16. Mayer P, Mayer B, Mayer G. Systems biology: building a useful model from multiple markers and profiles. Nephrol Dial Transplant 2012; 27: 3995-4002.

17. Craddock C, Jilani N, Siddique S, Yap C, Khan J, Nagra $S$, Vyas $P$. Tolerability and clinical activity of posttransplantation azacitidine in patients allografted for acute myeloid leukaemia treated on the RICAZA trial. Biol Blood Marrow Transplant 2016; 22: 385-390.

18. de Lima MC, da Silva DB, Freund APF, Dacoregio JS, Costa TEJB, Costa I, Silva ML. Acute Myeloid Leukaemia: analysis of epidemiological profile and survival rate. http://dx.doi.org/10.1016/j.jped.2015.08.008. 2016.

19. Liu S, Wang W, Zhou X, Ding Z, Gu R. A 2-DE-based proteomic study on the toxicological effects of cisplatin in L02 cells. Environ Toxicol Pharmacol 2015; 39: $167-$ 175.

20. Radic-Sarikas B, Rix U, Stukalov A, Gridling M, Müller $A C$, Colinge J, Bennett $K L$. Enhancing cognate target elution efficiency in gel-free chemical proteomics. EuPA Open Proteom 2015; 9: 43-53.

21. Ruvolo PP, Qiu Y, Coombes KR, Zhang N, Neeley ES, Ruvolo VR, Kornblau SM. Phosphorylation of GSK3a/ $\beta$ correlates with activation of $A K T$ and is prognostic for poor overall survival in acute myeloid leukaemia patients. BBA Clin 2015; 4: 59-68.

22. Quintás-Cardama A, Zhang N, Qiu YH, Post S, Creighton CJ, Cortes J, Kornblau SM. Loss of TRIM62 expression is an independent adverse prognostic factor in acute myeloid leukaemia. Clin Lymphoma Myeloma Leuk 2015; 15: 115-127.

23. Puissant A, Fenouille N, Alexe G, Pikman Y, Bassil CF, Mehta $S$, Kung AL. SYK is a critical regulator of FLT3 in acute myeloid leukemia. Cancer Cell 2014; 25: 226-242.

24. Banerjee J, Nilsen-Hamilton M. Aptamers: multifunctional molecules for biomedical research. J Mol Med (Berl) 2013; 19: 1333-1342.

25. Xing H, Hwang K, Li J, Torabi SF, Lu Y. "DNA aptamer technology for personalized medicine. Curr Opin Chem Eng 2014; 4: 79-87.

26. Zhao N, Pei SN, Qi J, Zeng Z, lyer SP, Lin P, Zu Y. Oligonucleotide aptamer-drug conjugates for targeted therapy of acute myeloid leukemia. Biomaterials 2015; 67:42-51.

27. Strassberger V, Gutbrodt KL, Krall, N, Roesli C, Takizawa H, Manz MG, NeriD. A comprehensive surface proteome analysis of myeloid leukemia cell lines for therapeutic antibody development. J Proteomics 2014; 99: 138-151.

28. Roesli C, Borgia B, Schliemann C, Gunthert M, WunderliAllenspach $H$, Giavazzi $R$, Neri $D$. Comparative analysis of the membrane proteome of closely related metastatic and non-metastatic tumor cells. Cancer Res 2009; 69: 5406-5414.

29. Xiong $Q$, Yang $Y$, Wang $H$, Li J, Wang S, Li Y, Hu, S. Characterization of miRNomes in acute and chronic myeloid leukaemia cell lines. Genomics Proteomics Bioinformatics. 2014; 12: 79-91.

30. Ruvolo PP, Ruvolo VR, Jacamo R, Burks JK, Zeng Z, Duvvuri SR, Yoo, SY. The protein phosphatase $2 A$ regulatory subunit B55a is a modulator of signaling and microRNA expression in acute myeloid leukaemia cells. Biochim Biophys Acta 2014; 1843: 1969-1977.

31. Frantzi $M$, Bhat A, Latosinska A. Clinical proteomic biomarkers: relevant issues on study design \& technical considerations in biomarker development. Clin TransI Med 2014; 3(7): b192.

32. Zhang L, Wen Q, Mao HP, Luo N, Rong R, Fan JJ, Yu $X Q$. Developing a reproducible method for the highresolution separation of peritoneal dialysate proteins on 2-D gels. Protein Expr Purif 2013; 89: 196-202.

33. Hourigan CS, Karp JE. Minimal residual disease in acute myeloid leukaemia. Nat Rev Clin Oncol 2013; 10: 460471.

34. Weber C, Schreiber TB, Daub H. Dual phosphoproteomics and chemical proteomics analysis of erlotinib and gefitinib interference in acute myeloid leukemia cells. J Proteomics 2012; 75: 1343-1356.

35. Buchi F, Spinelli E, Masala E, Gozzini A, Sanna A, Bosi A, Santini V. Proteomic analysis identifies differentially expressed proteins in AML1/ETO acute myeloid 
leukaemia cells treated with DNMT inhibitors azacitidine and decitabine. Leuk Res 2012; 36: 607-618.

36. Hollenbach PW, Nguyen AN, Brady H, Williams M, Ning $Y$, Richard N, MacBeth KJ. A comparison of azacitidine and decitabine activities in acute myeloid leukaemia cell lines. PLoS One 2010; 5: e9001.

37. Qiu X, Hother C, Ralfkiaer UM, Sogaard A, Lu Q, Workman CT, Liang G, Jones PA, Grønbæk K. Equitoxicdoses of 5-azacytidine and 5-aza-
2'Deoxycytidine induce diverse immediate and overlapping heritable changes in the transcriptome. PLoS One 2010; 5: e12994.

38. Flotho C, Claus R, Batz C, Schneider M, Sandrock I, Ihde $S$, Lübbert M. The DNA methyltransferase inhibitors azacitidine, decitabine and zebularine exert differential effects on cancer gene expression in acute myeloid leukaemia cells. Leukaemia 2009; 23: 1019-1028. 